\title{
Surface Modified Ni Nanoparticles Produced by the Electrical Explosion of Wire
}

\author{
Alexander P. Safronov ${ }^{1,2, a}, \underline{\text { Galina V. Kurlyandskaya }{ }^{1,3, b,},}$ \\ Satindar M. Bhagat ${ }^{4, c}$, Igor V. Beketov ${ }^{2}$, Aidar M. Murzakaev ${ }^{2}$, Iñaki Orue ${ }^{3}$, \\ Aitor Larrañaga ${ }^{3}$ \\ ${ }^{1}$ Ural Federal University, 620002, Ekaterinburg, Russia \\ ${ }^{2}$ Institute of Electrophysics UD RAS, 620016, Ekaterinburg, Russia \\ ${ }^{3}$ University of the Basque Country UPV-EHU, Faculty of Science and Technology, 48940, Leioa, \\ Spain \\ ${ }^{4}$ University of Maryland, 20742, College Park, USA \\ a email: safronov@iep.uran.ru, b, e-mail : galina@we.Ic.ehu.es, ce-mail: bhagat@physics.umd.edu
}

Keywords: Magnetic nanoparticles, surface modification, aromatic solvents, ambient conditions

\begin{abstract}
Spherical nickel nanoparticles were prepared by the electrical explosion of wire. The asprepared nanoparticles were modified immediately after fabrication at room temperature in order to provide tunable surface properties with focus on the development of composites filled with nanoparticles. Following liquid modificators were used: hexane, toluene and the solution of polystyrene in toluene. In one case the surface modification by carbon was made in gas phase as a result of hydrocarbon injection. The average size of the nanoparticles was about $50 \mathrm{~nm}$ and unit cell parameters were close to $0.351 \mathrm{~nm}$. Detailed characterization was done by X-ray diffraction, transmission electron microscopy, and magnetization measurements. Sphericity was also checked using microwave resonant absorption.
\end{abstract}

\section{Introduction}

Fully separated magnetic nanoparticles (MNPs) are being intensely studied with focus on technological applications [1-3]. High specific area is an important parameter because it provides enhanced interaction at the phase boundaries and contributes to the level of chemical interacions on the surface. Chemical surface properties of MNP strongly depend on the fabrication technology. Although methods based on the high energy dispersion and metal condensation in an inert gas provide metal surfaces of the as-prepared nanomaterial, MNPs oxidation upon exposure to the ambient oxygen is inevitable. It causes changes in chemical interactions on the surface. The last fact must be taken into account for the design of nanocomposites with embedded MNPs. One of the efficient methods providing very high production rate (of order of $200 \mathrm{~g} / \mathrm{h}$ ) is the electrical explosion of wire (EEW) [1]. In this work we have prepared nickel MNPs by the EEW technique. The as-prepared MNPs were surface modified in order to provide tunable surface properties. Their structure, morphology, magnetic and microwave properties were comparatively analyzed.

\section{Experiment}

Nickel nanoparticles were prepared by the electrical explosion of wire. The as-prepared MNPs were modified immediately after fabrication at room temperature in order to provide tunable surface properties with focus on the development of MNPs containing composites. Following liquid modifiers were used: hexane (Ni-I), toluene (Ni-II) and the solution of polystyrene in toluene (NiIII). In Ni-IV the surface was covered by the deposited carbon layer as a result of hydrocarbon injection to gas phase of EEW apparatus (Table 1). The specific surface was determined by low temperature nitrogen adsorption: mean diameter of about $50 \mathrm{~nm}$ was obtained in all cases under consideration. Transmission electron microscopy was performed using JEOL JEM2100 microscope. 
Magnetic measurements at room and cryogenic temperatures were done using Physical Property Measurement System Quantum Design (PPMS). Microwave resonant absorption, ferromagnetic resonance (FMR) and zero-field absorption were studied by the microwave techniques based on usage of a conventional homodyne spectrometer for the frequency of $8.81 \mathrm{GHz}$. A sample was located in the cavity such way that the radio frequency electric field was nearly zero. An external magnetic field $(\mathrm{H})$ and microwave magnetic field $\left(\mathrm{h}_{\mathrm{rf}}\right)$ were either perpendicular to each other $\mathrm{H} \perp$ $\mathrm{h}_{\mathrm{rf}}\left(\alpha=0^{\circ}\right)$ for FMR or parallel to each other for microwave absorption $\left(\alpha=90^{\circ}\right)$. The widths were large and therefore field modulation was not used.

X-Ray diffraction (XRD) measurements were done using a PHILIPS X'PERT PRO diffractometer, operating at $40 \mathrm{kV}$ and $40 \mathrm{~mA}$ with $\mathrm{Cu}-\mathrm{K}_{\alpha}$ radiation $(\lambda=1.5418 \AA)$ and a PIXcel solid state detector. A fixed divergence and antiscattering slit giving a constant volume of sample illumination were used. The X-ray powder diffraction data were used for preliminary identification of the initial compound and the possible formation of oxides were evaluated using the Powder Diffraction File database. In all the cases the obtained signal can be assigned to metallic $\mathrm{Ni}$, the deconvolution of the initial (111) diffraction maxima (at $\sim 44.9^{\circ}$ in $2 \theta$ ) provides an indication of the number of phases present in each XRD diagram. This procedure was carried out using the peak-fit option of the WinPLOTR program without structural model (Figure 1). The simulated profiles were then used to recalculate the starting unit cell parameters from the $2 \theta$ peak positions. The information from broadened X-ray diffraction lines is normally used to estimate the average size of coherent diffraction domains by using the Scherrer approach: $L_{h k l}=K \cdot \lambda / \beta_{h k l} \cdot \cos \theta, \mathrm{L}_{\mathrm{hkl}}$ is the crystal size, $\mathrm{K}$ is the particle shape factor, $\lambda$ is the X-ray wavelength, $\beta_{\mathrm{hkl}}$ is the broadening of the diffraction line measured at half the line maximum intensity and $\theta$ is the diffraction angle. The broadening of the peak is related to the crystal size and to the instrumental contribution provided by the used equipment conditions: $\beta_{h k l}=\left(B_{h k l}-b\right)$, where $\mathrm{B}_{\mathrm{hkl}}$ is the half width parameter measured on the X-ray pattern by the deconvolution of the first diffraction maxima and $\mathrm{b}$ is the broadening derived from the instrumental resolution.

\section{Results and discussions}

Fig. 1 and Table 1 summarize the results of the structural studies. Core-shell structure (pure Ni core and solid solution of $\mathrm{C}$ in $\mathrm{Ni}$ for shell) is clearly observed for Ni-IV sample. Quite in accordance with the structural studies the saturation magnetization (Fig. 2) of the Ni-VI sample is the smallest one as to expect for the smallest crystallite size. At room temperature the magnetization curves are close to saturation at reasonably low magnetic fields: for all MNPs the magnetization in the field of $18 \mathrm{kOe}$ insured the 0.98 value of the saturation magnetization. Using the saturation magnetization approach for Ni-II MNPs we obtained $\mathrm{Ms} \approx 49 \mathrm{emu} / \mathrm{g}$ for $\mathrm{T}=300 \mathrm{~K}$, which is very close to the one obtained directly from the experimental $\mathrm{M}(\mathrm{H})$ dependence and only about $5 \%$ lower than the expected for bulk fcc $\mathrm{Ni}$ value $\mathrm{Ms} \approx 55 \mathrm{emu} / \mathrm{g}$ [4].

Table 1. Sample description and selected structural properties

\begin{tabular}{|c|c|c|c|c|c|c|c|}
\hline Sample & $\begin{array}{l}\text { Diffraction angle } \\
(2 \theta) \text { for }(111)\end{array}$ & $\begin{array}{l}\text { d-spacing } \\
(\AA)\end{array}$ & $\begin{array}{l}\text { Unit cell } \\
\text { parameter }(\AA)\end{array}$ & $\begin{array}{l}\text { Cryst. } \\
\text { size(nm) }\end{array}$ & Liquid & $\begin{array}{l}\mathrm{H}_{\text {res }} \\
(\mathrm{kOe})\end{array}$ & $\begin{array}{l}\Delta \mathrm{H} \\
(\mathrm{kOe})\end{array}$ \\
\hline $\mathrm{Ni}-\mathrm{I}$ & $44.687(2)$ & $2.026(1)$ & $3.509(1)$ & $36(3)$ & Hexane & $\begin{array}{c}2.7 \\
(0.2)\end{array}$ & $\begin{array}{c}2.6 \\
(0.2)\end{array}$ \\
\hline Ni-II & $44.666(2)$ & $2.027(1)$ & $3.511(1)$ & $34(3)$ & Toluene & $\begin{array}{c}2.7 \\
(0.2)\end{array}$ & $\begin{array}{c}2.6 \\
(0.2)\end{array}$ \\
\hline Ni-III & $44.750(3)$ & $2.023(1)$ & $3.504(1)$ & $29(3)$ & Toluene & $\begin{array}{c}2.7 \\
(0.2)\end{array}$ & $\begin{array}{c}2.6 \\
(0.2)\end{array}$ \\
\hline $\begin{array}{l}\text { Ni-IV with } \\
\text { C-covering }\end{array}$ & $44.28(1)$ & $2.044(1)$ & $3.540(1)$ & $\begin{array}{c}11(2) \\
28(3)\end{array}$ & Hexane & $\begin{array}{c}2.4 \\
(0.2)\end{array}$ & $\begin{array}{c}2.7 \\
(0.2)\end{array}$ \\
\hline
\end{tabular}




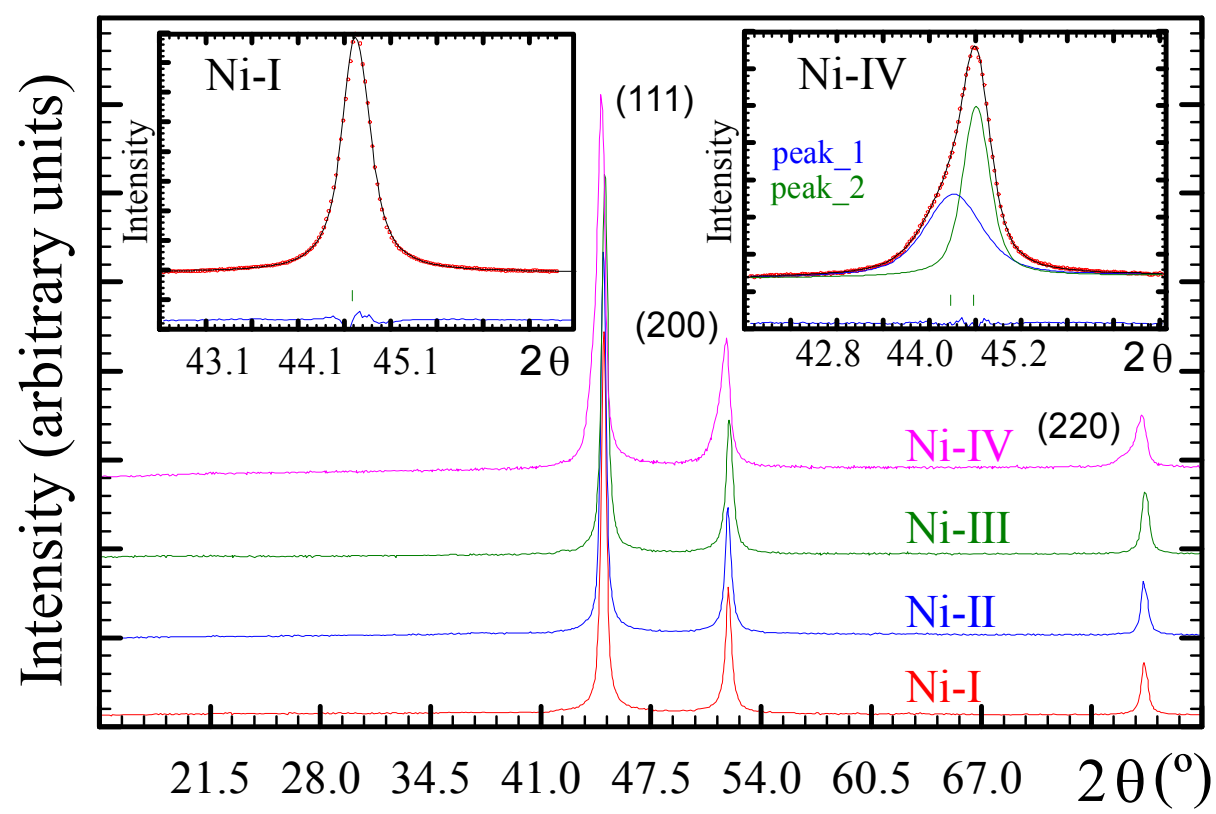

Fig. 1. X-ray powder diffraction patterns including (hkl) Miller Indexes for the EEWNi MNPs. Insets show (111) peak deconvolutions for Ni-I and Ni-IV MNPs.
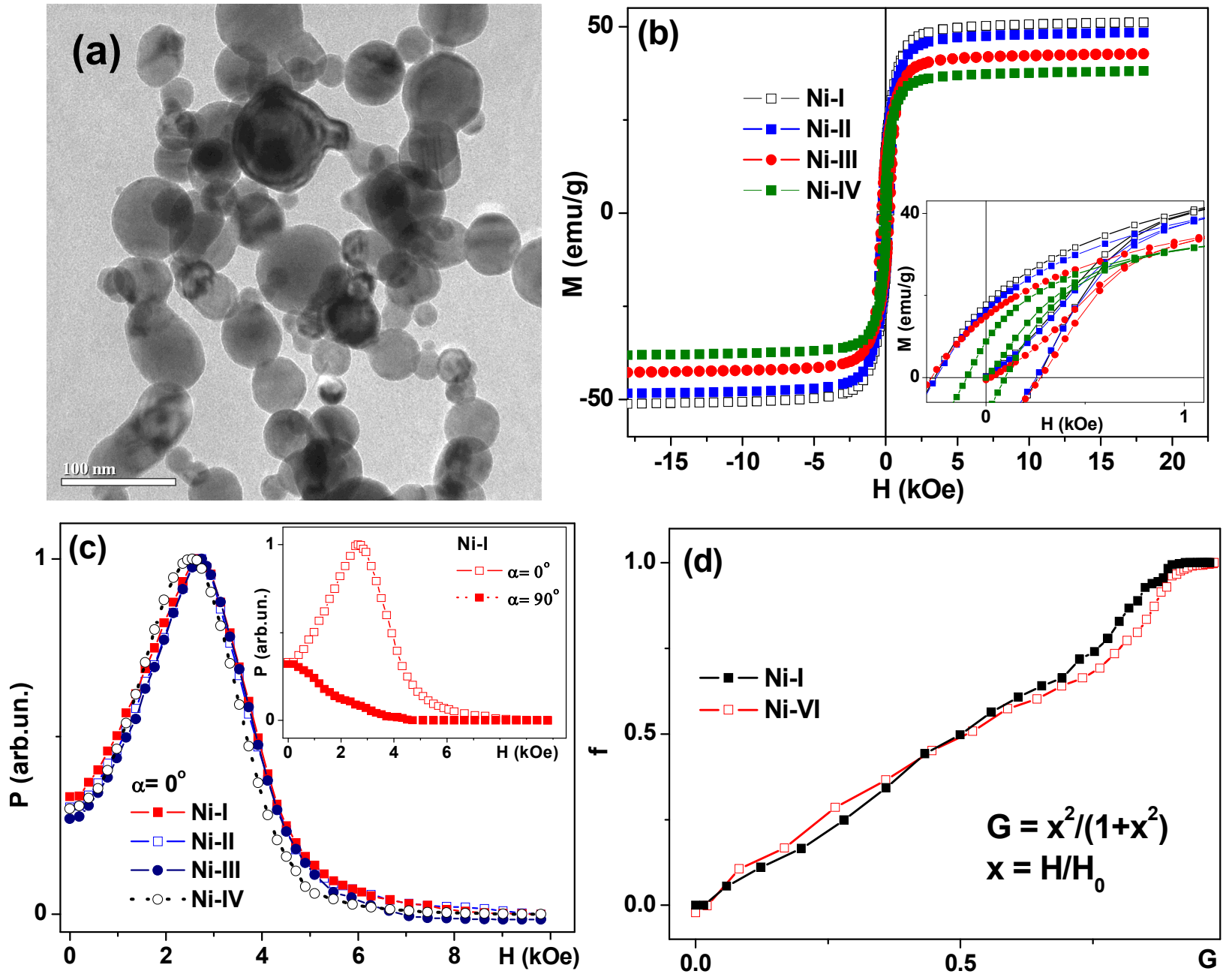

Figure 2. TEM image of Ni MNPs (a); hysteresis loops measured at $300 \mathrm{~K}$ for Ni MNPs, inset shows low field behaviour (b). FMR $\left(\alpha=0^{\circ}\right)$; inset shows examples for $\mathrm{H} \perp \mathrm{h}_{\mathrm{rf}}$ and $\mathrm{H} \| \mathrm{h}_{\mathrm{rf}}\left(\alpha=90^{\circ}\right)$ (c). Empirical function $\mathrm{G}$ and figure of merit $\mathrm{f}=(\mathrm{P}(\mathrm{H}=0)-\mathrm{P}(\mathrm{H})) / \mathrm{P}(\mathrm{H}), \mathrm{H}_{0}$ is the value of external field where the absorption falls to half of its value at the zero-field: $\mathrm{H}_{0}=1.57 \mathrm{kOe}$ for $\mathrm{Ni}-\mathrm{I}$ and $\mathrm{H}_{0}=1.37 \mathrm{kOe}$ for Ni-IV cases (see also Ref. [4]). 
FMR measurements indicate that all MNPs are reasonably uniform magnetically homogeneous materials: only one resonance line was observed (Fig. 2). Resonance fields $\left(\mathrm{H}_{\mathrm{res}}\right)$ and linewidths $(\Delta \mathrm{H})$ are listed in Table 1. For spherical Ni MNPs the resonance field can be described by equation $\omega / \gamma=\mathrm{H}_{\text {res }}$, where $\omega=2 \pi \mathrm{f}$ is the microwave frequency and $\gamma$ is the gyromagnetic ratio $(\gamma=8.8 \times \mathrm{g}$ ( $\mathrm{Mrad} / \mathrm{Oe}), \mathrm{g}$ - spectroscopic Landé factor). We obtained $\mathrm{g} \approx 2.18$, which is very close to the value previously reported for nickel [5]. In Ni-I, Ni-II and Ni-III samples the applied field for resonance lies very close to the one given by above mentioned equation. In the case of Ni-IV sample the experimental resonance field lies below $\mathrm{H}=2.7 \mathrm{kOe}$, indicating the presence of the internal fields causing this shift. The observed shortfall of about $0.3 \mathrm{kOe}$ can be understood by invoking stresses created by the carbon containing shell with different structural features. Surface passivation in hexane, toluene and polystyrene solution - Ni-I, Ni-II and Ni-III cases does not significantly change microwave properties of MNPs and therefore these treatments can be viewed as promising step of fabrication of Ni MNPs based composites. In all cases sizable zero field absorption was evident which is not surprising for metallic MNPs with high conductivity [3]. This signal is a consequence of a large dynamic magnetic permeability, it originated from the spins and it is not related to magnetoresistance of conduction electrons. Resonant and zero-field absorption overlapped making the quantitative analysis of the signals somehow difficult. Even so the description of low-field behaviour by the empirical relation proposed previously (Ref. [3]) gave quite satisfactory results in the case of a new kind of material - EEW MNPs. Fig.2d shows two examples of such an analysis.

\section{Summary}

Spherical nickel MNPs were prepared by the electrical explosion of wire technique. Their surfaces were modified immediately after fabrication at room temperature by following liquid modifiers: hexane (Ni-I), toluene (Ni-II) and the solution of polystyrene in toluene (Ni-III). In Ni-IV the surface covering consisted of carbon solution in $\mathrm{Ni}$ as a result of hydrocarbon injection. All MNPs exhibited a sizable loss at a zero-field. The position of the FMR corresponds well to that one expected for spherical nickel MNPs $\left(\mathrm{H}_{\text {res }} \approx 2.7 \mathrm{kOe}\right)$ in all cases except N-IV. For N-IV case $\mathrm{H}_{\text {res }} \approx 2.4 \mathrm{kOe}$. The observed shortfall of about $0.3 \mathrm{kOe}$ can be understood by invoking stresses created by the carbon containing shell. Experiments are underway to make these particles useful for applications.

\section{Acknowledgements}

This publication is based on work supported by a grant CRDF - UB RAS RUE2-7103-EK-13 from the U.S. Civilian Research \& Development Foundation (CRDF Global) with funding from the United States Department of State. The opinions, findings and conclusions stated herein are those of the authors and do not necessarily reflect those of CRDF Global or the United States Department of State. Selected measurements were performed at SGIker services of UPV-EHU.

\section{References}

[1] G.V. Kurlyandskaya, S.M. Bhagat, A.P. Safronov, I.V. Beketov and A. Larrañaga, AIP Advances, 1 (2011) p. 042122.

[2] A.P. Safronov, G. V. Kurlyandskaya, A.A. Chlenova, M.V. Kuznetsov, D.N. Bazhin, I.V. Beketov, M.B. Sanchez-Ilarduya, and A. Martinez-Amesti, Langmuir 30 (2014) p. 3253.

[3] V.V. Srinivasu, S.E. Lofland, S.M. Bhagat, K. Ghosh, S.D. Tyagi, J. Appl. Phys. 86 (1999) p. 1067.

[4] B.D. Cullity, Introduction to Magnetic Materials, Addison-Wesley Publishing Company, 1972. pp. 233-236.

[5] N. Bloembergen, Phys. Rev. B, 78, (1950) p. 572. 